\title{
ARQ Scheme of Multimedia Streaming for Hybrid Delivery over Heterogeneous Network
}

\author{
Sung Hei Kim*, Chang Kyu Lee**, Jae Hyoung Lee**, Shin Gak Kang* \\ *ETRI(Electronics and Telecommunications Research Institute), Rep. of Korea \\ **UST(University of Science and Technology), Rep. of Korea \\ shkim@etri.re.kr, echkyu@etri.re.kr, hafins@etri.re.kr, sgkang@etri.re.kr
}

\begin{abstract}
Nowadays, many mobile devices have multiple networking modules to receive data from various networks such as WI-FI, 3G, Wimax, DVB-H, etc. The mobile devices are used to receive multimedia streams through various networking interfaces. However, mobile characteristics can cause loss of packets and needs an effective method to recover the loss to provide high-quality multimedia streaming service. ARQ (Automatic Repeat Query) can be used to re-receive the loss packets that are being delivered. The heterogeneous networks can be used to effectively deliver multimedia stream and also provides ARQ to recover the loss packets. The retransmitted data can be delivered over bi-directional network such as Internet. This paper proposes a hybrid delivery for heterogeneous network environment and efficient ARQ that can be considered for multimedia streaming.
\end{abstract}

Keywords - Multimedia streaming, Hybrid delivery, heterogeneous networks, Automatic Repeat Query

\section{INTRODUCTION}

Television broadcasting via terrestrial and satellite transmission channels has been the main means for multimedia delivery[1]. Many television broadcasting contents delivered to the mobile devices, but has faced much loss of packets due to the mobile characteristics. For many contents, the user does not care about small delays in viewing the multimedia streaming. However, user has high expectation for smooth and clear view of the multimedia contents. Most of the mobile device has multiple networking modules. The networks can be used to receive data in broadcasting mode or interactive mode. The broadcasting mode is used to deliver data to multiple devices, such network can be terrestrial and satellite network. The interactive mode is used to deliver data between two devices; such network can be Internet, IP (Internet Protocol) - based point-to-point communications. It is possible to combine to two modes of communication to enable advanced and sophisticated multimedia services.

Automatic Repeat Query (ARQ), is an error-control method for data transmission that uses acknowledgements (messages sent by the receiver indicating that it has correctly received a data frame or packet) and timeouts (specified periods of time allowed to elapse before an acknowledgment is to be received) to achieve reliable data transmission over an unreliable network. If the sender does not receive an acknowledgment before timeout, it usually re-transmits the frame/packet until the sender receives an acknowledgment or the window buffer exceeds the predefined number for retransmission [2].

ARQ can be used to re-receive the loss packets that have been delivered. The broadcasting networks can be used to effectively deliver multimedia stream and bi-directional network can be used to provide ARQ to recover the loss packets. Thus, multiple networks can be involved in the multimedia streaming service. This paper proposes a hybrid delivery for heterogeneous network environment and efficient ARQ that can be considered for multimedia streaming. There are many studies on the ARQ technique itself to provide better scheme for various environments. However, this paper focuses on ARQ scheme to compensate for the loss that can occur when providing broadcast service.

This paper is organized as follows. The following chapter describes some related works of this paper with the description on ARQ scheme and delivery environment of the heterogeneous networks. The chapter 3 describes the proposed ARQ scheme that makes use of the multimedia contents server. Finally, this paper ends in chapter 4 with the concluding remarks.

\section{RELATED WORKS}

\section{A. Automatic Repeat Query (ARQ) schemes}

According to Wikipedia, Automatic Repeat Query (ARQ), is defined as an error-control method for data transmission that uses acknowledgements and timeouts to achieve reliable data transmission over an unreliable service. If the sender does not receive an acknowledgment before the timeout, it usually retransmits the frame/packet until the sender receives an acknowledgment or the window buffer exceeds the predefined number for retransmission [1]. Although, there are much research on the topic of ARQ, the most typical ARQ schemes are Stop-and-wait ARQ, Go-Back-N ARQ, and Selective Repeat ARQ.

In Stop-and-wait ARQ scheme, sender sends one data packet at a time. After sending each data packet, the sender does not send any packet until it receives an acknowledgement 
signal from the receiver. The transmit window size and receive window size are 1[4].

In Go-Back-N ARQ scheme, sender continues to sends specified number of data packet without receiving an acknowledgement from the receiver. The transmit window size is $\mathrm{N}$ and receive window size is $1[5]$.

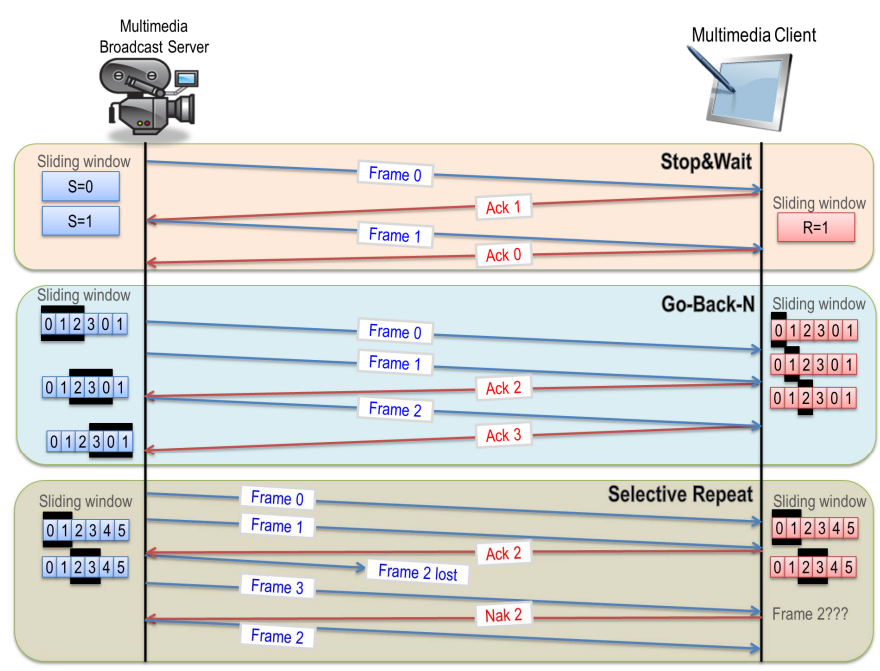

Figure 1. ARQ Flows

In Selective Repeat ARQ scheme, sender sends a number of data packet specified by a window size even without the need to wait for individual acknowledgement from the receiver. However, the receiver sends acknowledgement for each packet individually. The receiver accepts out-of-order frames and buffers them. The sender individually retransmits frames that have timed out.

ARQ is a common technique used to make a wireless link reliable [3]. Therefore, it is particularly needed for mobile devices, where frequent movement can cause temporary network disconnection and packet loss. Although the ARQ scheme is needed feature for wireless or mobile device, it can be used in any type of device or network that has a possibility of packet loss.

The ARQ scheme described in this paper is not dependent on the actual type of ARQ used. Any of the three types or any new types from the future can be used. The core function behind the ARQ schemes depends on the window size and acknowledgement method used. This paper provides a method for the sender to inform the receiver of the preferred process of ARQ scheme

\section{B. Delivery environment of the heterogeneous network}

The typical heterogeneous network for multimedia delivery is shown in Figure 2.

Devices can be connected to more than one network and are capable of receiving multimedia contents over multiple network interfaces. A single streaming content can be delivered in the multiple networks. As shown in Figure 2. , typical transmission networks are broadcast network and Internet. The broadcast network is used to deliver live television or recorded contents to the multimedia devices such as TV, set-top box. The broadcast network is mostly used in a unidirectional manner, since it is used to deliver contents to countless number of receivers. The multimedia broadcast server can send multimedia stream to the various types of multimedia clients. The multimedia clients can be in various forms, wired, wireless, and mobile. Any device that can receive and consume the broadcasted multimedia is eligible to receive the broadcasted contents. Since the multimedia broadcast server delivers multimedia streaming to the broadcast network in the unidirectional manner. The multimedia client receives the contents in best effort manner. Figure 2. shows how the different types of multimedia client can receive multimedia contents though the use of broadcast network.

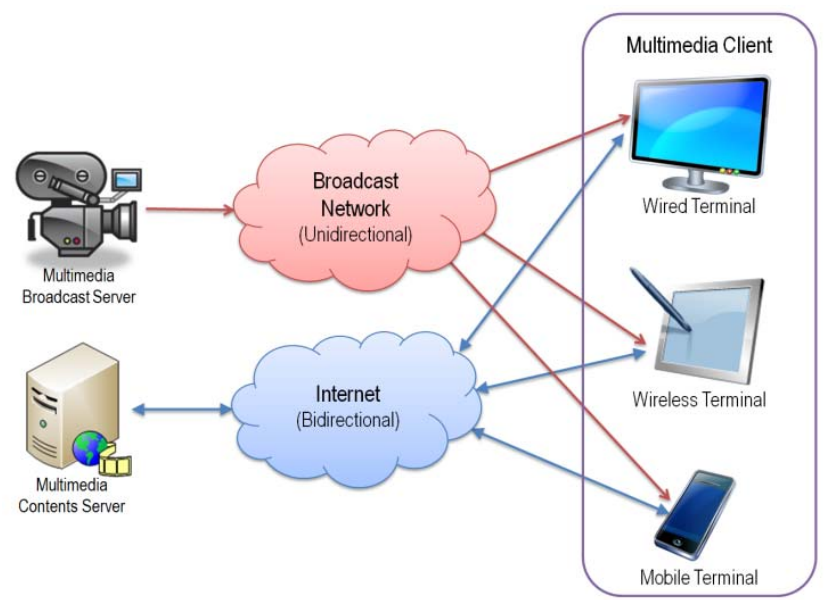

Figure 2. Multimedia Delivery over Heterogenous Network

The most widely used bidirectional network would be Internet, which can be used to provide various forms of interactive service. In this type of network, the receiver can make any type of request to the sender to satisfy its needs. It would be efficient to use the bidirectional network for broadcasting contents, since it can request the sender to retransmit any loss packet. However, the maximum number of receivers is limited for bidirectional network. This paper proposes a scheme to use the broadcast network to receive multimedia contents and use the Internet for ARQ in order to realize ARQ for any types of receiving devices in providing multimedia streaming.

\section{III.PROPOSED ARQ SCHEME}

This chapter describes the proposed ARQ scheme. The multimedia broadcast server sends multimedia stream to any network, such as broadcast network or Internet. In this case broadcast network is preferable, since the number of devices supported is limitless. The multimedia content server also receives the data from the multimedia broadcast server. The service provider will need to make sure the multimedia content server does not suffer from excessive packet loss. The retransmission request is performed between the multimedia client and multimedia contents server through the Internet.

As shown in Figure 3. multimedia broadcast server broadcasts the multimedia packet to the multimedia client and 
multimedia contents server. The client does not receive packet (222). Without an adequate retransmission scheme, client will have to play the multimedia contents without packet (222). In some case the packet (222) can be an important frame such as $\mathrm{i}$-frame, which is a piece of transmitted video with the $p$-frame and $b$-frame referencing it. The user will have to bear the broken images or temporary blackout when viewing the streaming contents. This phenomenon can occur frequently for the mobile devices where the point of attachment is not stable. If the service provider can provide a method to provide ARQ, such problem would decrease and clear picture can be provided at the client side.

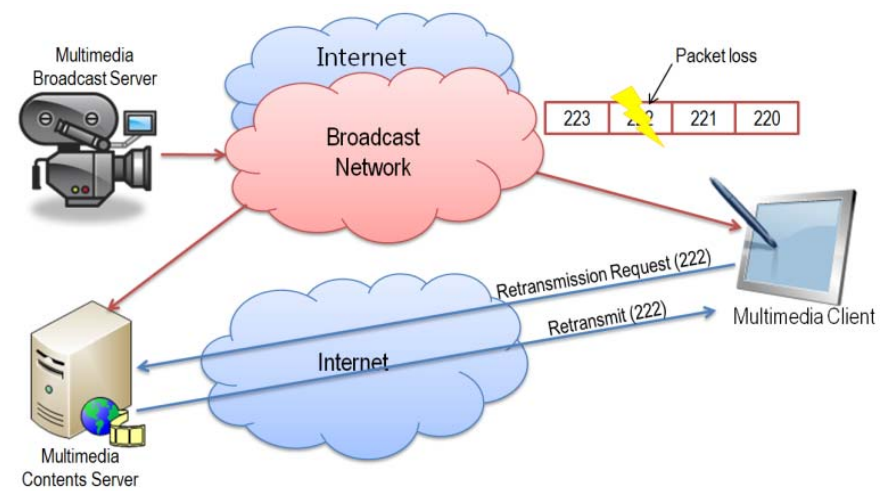

Figure 3. ARQ in the Heterogenous Network

As shown in Figure 3. multimedia broadcast server broadcasts the multimedia packet to the multimedia client and multimedia contents server. The client does not receive packet (222). Without an adequate retransmission scheme, client will have to play the multimedia contents without packet (222). In some case the packet (222) can be an important frame such as $\mathrm{i}$-frame, which is a piece of transmitted video with the $\mathrm{p}$-frame and b-frame referencing it. The user will have to bear the broken images or temporary blackout when viewing the streaming contents. This phenomenon can occur frequently for the mobile devices where the point of attachment is not stable. If the service provider can provide a method to provide ARQ, such problem would decrease and clear picture can be provided at the client side.

The packet retransmission is provided by the multimedia contents server. Although the packet is received from the other network, such as broadcast network, the client can perform ARQ protocol with the multimedia contents server. The server itself can provide the loss packet automatically, or the client can specifically request for the loss packet. This is dependent upon the type of ARQ scheme used. However, this paper proposes a scheme to use different server for different purpose.

\section{A. Allocation of multimedia contents server}

Since the multimedia broadcast is broadcasted to countless number of clients, it would be impossible for a single multimedia content server to support every receiving client.

As shown in Figure 4. multimedia broadcast server can have multiple sets of multimedia content server to provide
ARQ and retransmission to the clients. The clients can be gathered into a different group with support from the designated multimedia content server. The method of allocating the multimedia content server to a particular group is service dependent. However, grouping of the devices based on geographical level is one option that can be considered.

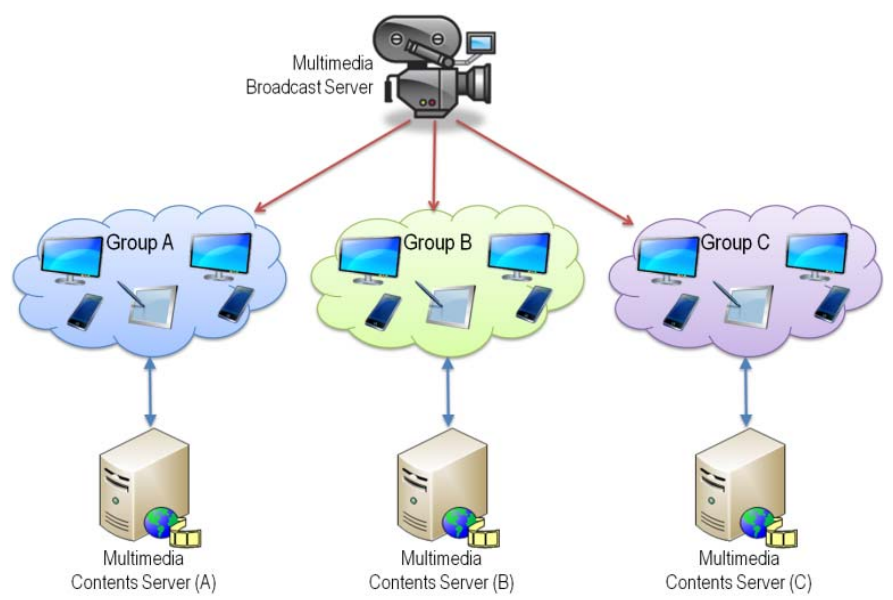

Figure 4. Allocating Multimedia Contents Server

\section{B. Retransmission flow}

The details of the retransmission flow are shown in Figure 5. The example ARQ mechanism used in this chapter is negative acknowledgement scheme, in which the client does not send acknowledgement, but sends negative acknowledgement to the server upon occurrence of packet loss. The multimedia client can request for the media to the multimedia broadcast server. This process can be omitted, since the broadcast server will be broadcasting the media with or without any receivers. It may not need to know of the existence of the receivers. The client can get the information of the media through other route such as Web page or EGP, that provides information on how the retrieve such media. The multimedia broadcast server will need to provide media configuration information to the client. The multimedia broadcast server may or may not explicitly send the media configuration related information to the requesting client. Normally, the multimedia broadcast server periodically sends media configuration information, and the client can start consuming the multimedia stream after receiving such information. The information regarding ARQ configuration can be delivered through this message, or it can be retrieved through other means such as webpage.

After receiving ARQ configuration information, client can connect to the multimedia contents server. The multimedia contents server can be used for single media streaming or multiple media streaming depending upon how the multimedia contents server and multimedia broadcast server are configured. However, the client can perform ARQ or retransmission for media data loss.

The client will be receiving the media data from the broadcast server. If it perceives packet loss, it will need to start retransmission. Before requesting for loss packet, it will need to calculate ARQ processing time. The ARQ processing 
time includes transmission time and ARQ processing time. Since the packet is a real-time data, the lifespan of the packet is short. It will need to consume the media with or without the loss packet after certain period of time. If the client observes that the expected consumption time of the loss packet is shorter that the ARQ processing time, it does not request for retransmission of media data. Upon reception of the retransmission request, the server also makes similar calculation for the same purpose.

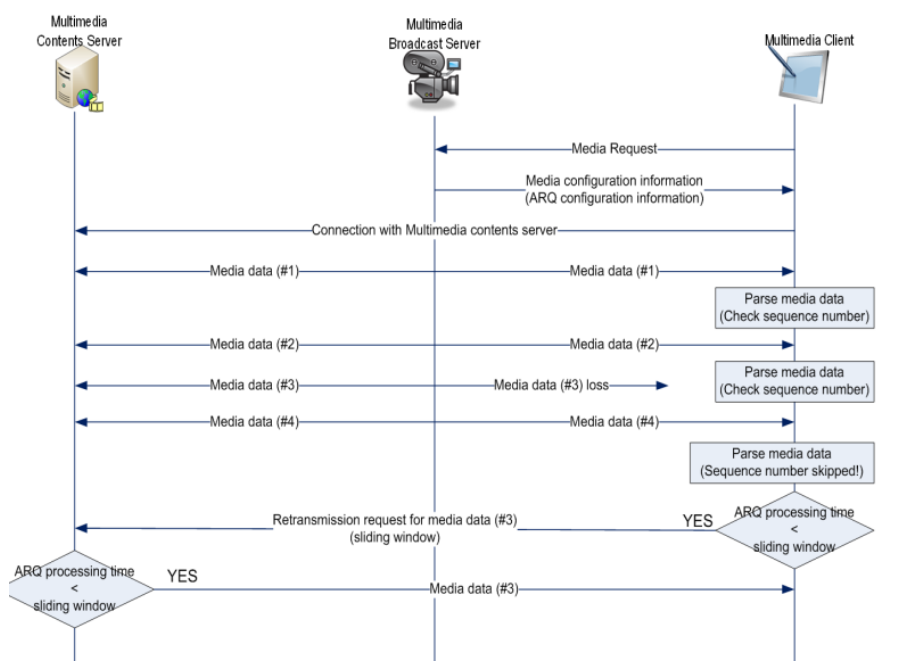

Figure 5. Retransmission Flow

\section{ARQ configuration information}

The following lists some of the information that can be delivered in the ARQ configuration message.

- Multimedia Contents Server address: network address of the Multimedia Contents Server. If Internet is used, it would be IPv4 or IPv6 address.

- $\quad$ ARQ port number: Layer 3 port number for performing ARQ protocol and retransmission.

- Multimedia Contents Server window size: window size of the Multimedia Contents Server. This is used to inform the client when it should make ARQ acknowledgement. If ARQ acknowledgement is not used, this information is not needed.

- Sliding window timeout: retransmission timeout. The Multimedia Contents Server will maintain the multimedia packet in buffer until this timeout

Note that the Multimedia Contents Server does not need to know the window size of the client. More information can be added depending upon the actual type of ARQ scheme used. However, the currently listed information is adequate to support any three of the popular ARQ schemes, i.e., Stop-andwait ARQ, Go-Back-N ARQ, and Selective Repeat ARQ.

\section{IV.CONCLUSION}

This paper proposes ARQ scheme that can be used in multimedia streaming service delivered over heterogeneous network. The ARQ scheme proposed is not dependent on the actual type of ARQ used. Any existing types or any new types of ARQ scheme from the future can be used with the proposed scheme. The proposed scheme can use the multimedia broadcast server in broadcasting multimedia contents, and multimedia contents server for ARQ protocol and retransmission. This paper provides detailed description on the retransmission flow for the proposed scheme. This paper also proposes some information that can be included in the ARQ configuration message.

\section{ACKNOWLEDGMENT}

This research is supported by the ICT Standardization program of MSIP (Ministry of Science, ICT \& Future Planning).

\section{REFERENCES}

[1] MPEG requirements groups, "Use Cases for MPEG Media Transport (MMT)”, N11542, July 2010

[2] Wikipedia, "ARQ", October 2012

[3] Ilmu Byun and Kwang Soon Kim, "Cooperative Hybrid-ARQ Protocols: Unified Frameworks for Protocol Analysis", ETRI Journal, Vol 22, No. 5, October 2011.

[4] Cheng Guo, et.al., "Designing Energy Efficient Automatic Repeat Request Protocol in Wireless Sensor Networks", CHANTS'09, Sept. 2009.

[5] Wikipedia, "Stop-and-wait ARQ", June 2013

[6] Wikipedia, "Go-Back-N ARQ", Nov 2013

[7] Wikipedia, "Selective Repeat ARQ", June 2013

[8] MPEG Ad-hoc group, "Implementation details of reception quality feedback in MMT service", M 25150, April 2012.

[9] MPEG systems group, Text of ISO/IEC 23008-1 PDAM2 Header Compression and Cross Layer Interface, N13699, August 2013.

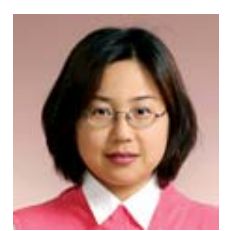

Sung Hei Kim received the B.S. and M.S. in computer science from Ehwa University in 1991 and from Chungnam University in 1995, respectively. She s pursing Ph.D degree in Chungnam University. She has been working as a researcher in ETRI since 1991. She is actively participating in standardization in ITU-T study group 11, 13, 16, ISO/IEC JTC 1/SC 6, MPEG, and W3C. Her topic of interests includes, multimedia delivery, peer-to-peer communication, multicasting, future network, and Internet routing.

Chang Kyu Lee received the B.S. in computer science and engineering from Inha University, Korea, in 2008. $\mathrm{He}$ is currently pursuing his M.S. and Ph.D. degree in engineering at University of science and technology, Korea. He is working for ETRI as UST student since 2008. He is actively participating in standardization in ITU-T study group 11, 13, ISO/IEC JTC 1/SC 6, MPEG. His research interests include peer-to-peer networking, multimedia communications.

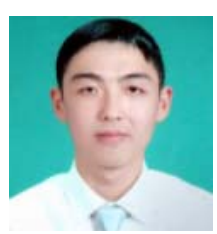

Jae Hyoung Lee received the B.E. degree from Kongju Univ. in 2010. He has received M.S. degree in broadband network engineer in University of Science and Technology in 2014. He is working in ETRI as UST student from 2011. His research interest includes service ontology, service oriented architecture, and service applications

Shin-Gak Kang received the B.S., M.S., and Ph.D in electronics engineering and information communication Chungnam University, in 1984, 1987, 1998, respectively. $\mathrm{He}$ is working for ETRI since 1984. Currently, he is a Director of media application standard research section. From 2008 he is a professor at the Department of Engineering, University of Science and Technology, Korea. $\mathrm{He}$ is actively participating in various international

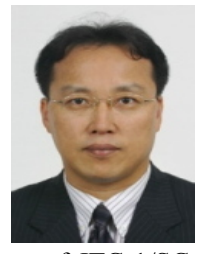
standard bodies as a Vice-chairman of ITU-T SG11, Convener of JTC 1/SC 6/WG 7, etc. His research interests include contents networking, multimedia communications, and Future Network. 